\title{
Demo Abstract: A Microphone Sensor based System for Green Building Applications
}

\author{
MD Abdullah Al Hafiz Khan, Sheung Lu, Nirmalya Roy, Nilavra Pathak \\ Department of Information Systems, Computer Engineering, University of Maryland Baltimore County \\ mdkhan1@umbc.edu, slu2@umbc.edu, nroy@umbc.edu, nilavra1@umbc.edu
}

\begin{abstract}
Acoustic sensing has influenced many applications in green building energy management, such as designing multi-modal energy disaggregation algorithms through fine-grained appliance state identifications or efficiently controlling the HVAC system based on the occupancy of the environment. In this demo paper we build a low-cost system prototype using off-the-shelf commercially available hardware (Raspberry $\mathrm{Pi}$ and super high gain microphone) to handle both acoustic sensing and its processing that is portable and easily deployable in any indoor environment. Our system is useful in detecting appliance noise for fine-grained energy metering and human voice for managing building energy footprint. We use the decibel strength of the sound to determine if it should be filtered out as a silence or stored in as an audio of interest. A fast fourier transform that quickly converts the sinusoidal input of the audio signals into its associated frequencies is implemented along with the Mel-Frequency Cepstral Coefficients (MFCCs) feature to distinguish between a human voice and an appliance noise. We also implement all the computations on-chip to quantify the energy-delay benefits.
\end{abstract}

\section{INTRODUCTION}

The use of sound to sense an environment is an effective approach for participatory sensing, smart home activity monitoring and intelligent building energy management applications. Detecting the number of occupants in an environment, their acoustic footprints along with their usage of appliances provide fine grained information for designing better solutions and data analytic techniques for green building energy management. We prototype an Acoustic Sensor Node (ASN) integrating Raspberry Pi (raspi) [2] and a super high gain microphone [4] that is able to detect audio signals in a midsize room. We choose these specific hardwares to make our Acoustic Sensor Node (ASN) both small in size and easily mountable in any indoor environment. The ASN can be used for smart-home applications to detect the use of appliances and associate them with specific human activities of daily living (ADLs) which not only promote energy-efficient building operations, but also provide unique insights into the context and lifestyle activities of individuals. We develop a prototype system meeting the needs of a wide variety of applications

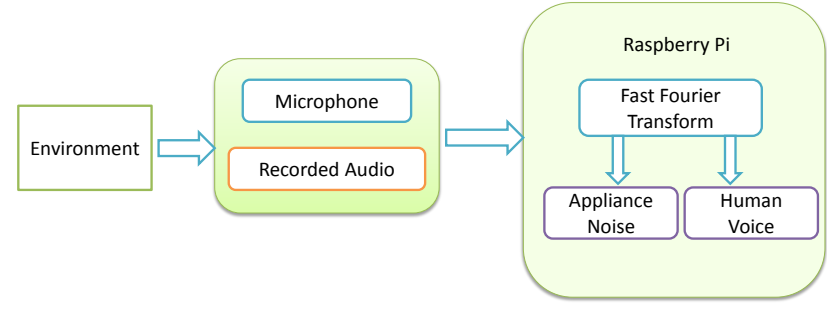

Fig. 1. Overall Architecture of Acoustic Sensor Node

which allows us to collect a multitude of data about the environment and its artifacts for developing monitoring, controlling and providing feedback solutions for green building environment.

\section{Overall Architecture}

The overall architecture of our proposed Acoustic Sensor Node prototype system has been shown in Fig. 1. Our acoustic sensor node consists of two logical parts: i) Audio signal detector and recorder, ii) Voice Separator. In the first module audio signal is detected and recorded via SoX [3] ( $\mathrm{SoX}$ is cross platform software tools able to record and convert audio to various format) and stored as WAV format to preserve the audio quality. It also detects silence and discard those portions. Files name are given based on the starting timestamps of specific audio file. The second module consists of algorithmic implementation (signal processing and feature vectors extraction) for calculating FFT [5] and MFCC [6]. After computing FFT, audio signal has been classified either as human conversations or background noises. The audio signal is then appropriately spliced and separated into categories of human voice, and appliance sound. The human conversation portions has been analyzed to detect the number of distinct voice threads in a specific room/zone.

\section{IMPLEMENTATION}

In our implementation audio signal is recorded via a Sound eXchange (SoX) script that automatically records 


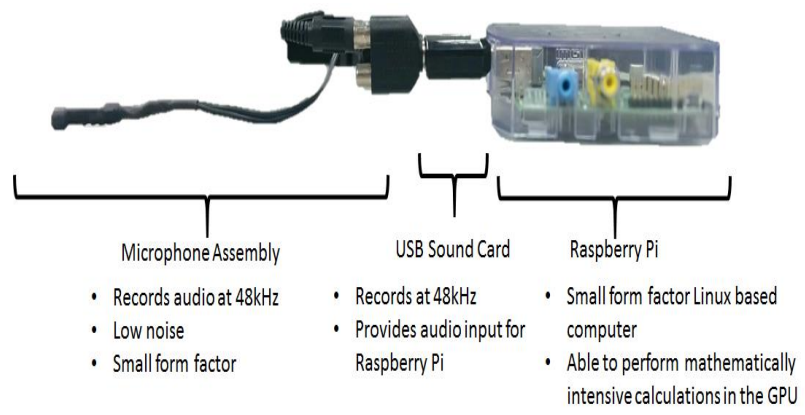

Fig. 2. ASN Hardware System

if there is a change of noise level such as from a silence to a non-silence. Pieces of the audio data is then passed through a Fast Fourier Transform (FFT) to identify between human voice and other noise. Human voice is distinct from the ambient noise as it usually produces sound between $300 \mathrm{~Hz}$ to $3000 \mathrm{~Hz}$ with a general distribution pattern across those frequencies.

The Raspberry Pi can perform mathematically intensive tasks like the FFT on the Graphics Processing Unit (GPU) thereby saving energy and computation time on the CPU. FFT library for the Raspberry Pi which consist of BCM2835 SoC V3D hardware [1] gives much better performance than the $700 \mathrm{MHz}$ ARM. We check the performance for different number of data points which is chosen as a power of $2(\mathrm{~N})$ [5]. The value of $\mathrm{N}$ is tested for $\mathrm{N}=8$ to 17 . The FFT has been used as a preliminary filter for audio signals as it is best suited for localized computation in our case. Extracted MFCC features has been used to distinguish between human voice and/or appliance noise. Our prototype system including Raspi and microphone sensor has been shown in Fig. 2.

\section{DEMO DESCRIPTION}

The proposed demo advocate the usability of an acoustic sensor node prototype for faster processing of acoustic noise on an ubiquitous hardware platform (Raspberry Pi) with a cheap off-the-shelf commercially available microphone sensor. An interface developed for the demo will plot real-time computational time required for GPU and CPU based audio signal processing technique using our prototype. The just-in-time performance of our ASN help improve designing acoustic signature enabled energy disaggregation algorithm, finegrained appliance state identification, abnormal power consumption event recognition, and building occupancy detection.

\section{RESUlts}

Our acoustic sensor node prototype posits that its Raspberry Pi's GPU FFT implementation is a viable

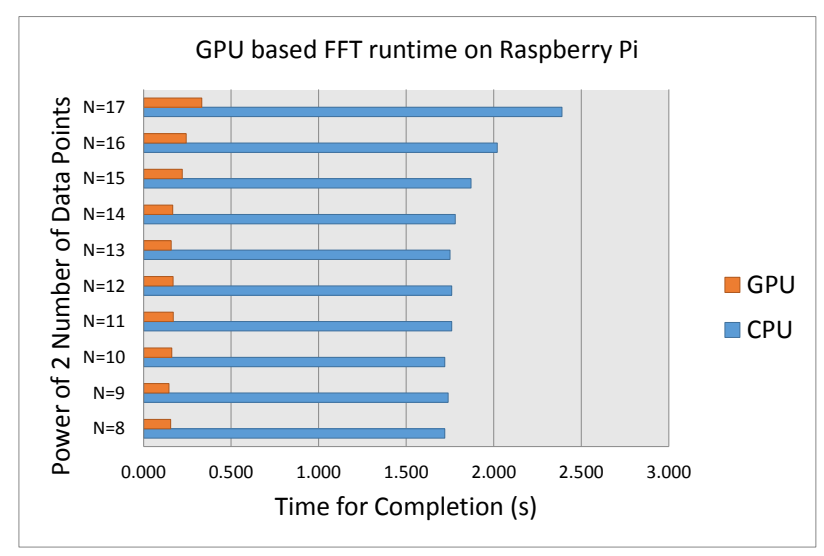

Fig. 3. FFT Computation Performance on GPU vs. CPU

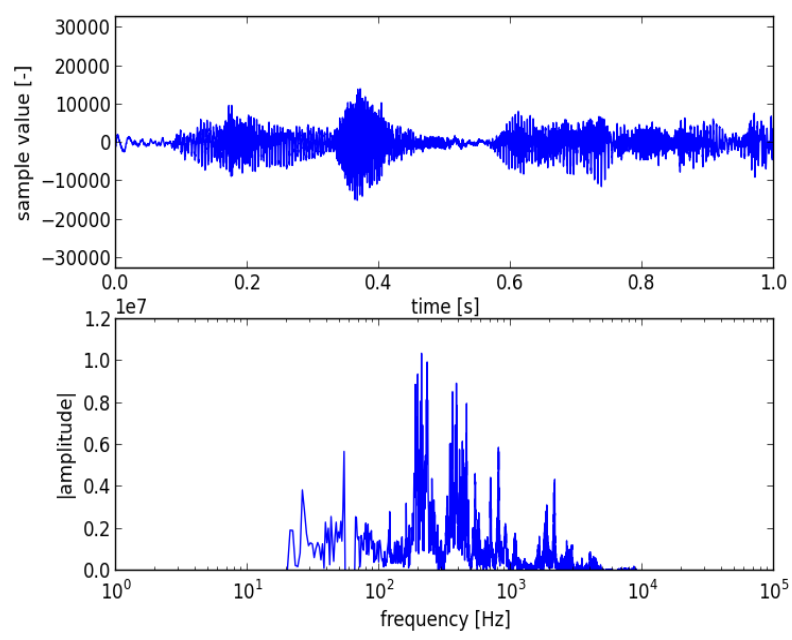

Fig. 4. Time domain and frequency domain representation of acoustic signal of human speech

solution for the ubiquitous deployment microphone sensor based system by reducing the energy consumption, computational resource usage while accelerating the faster processing. Fig. 3 shows GPU based FFT attest an order of magnitude (almost 10 times) better computational time than the CPU based FFT computation. Thus our ASN prototype implemented with GPU based FFT computation helps provide faster appliance state identification based on their acoustic signatures to design fine-grained energy disaggregation algorithms.

Fig. 4 represents the time domain vs. frequency domain acoustic signal of human speech. We can see that human voice ranges from $300 \mathrm{~Hz}$ to $5000 \mathrm{~Hz}$.

\section{CONCLUSIONS}

Acoustic sensing has many insightful and innovative applications, though often constrained by its implemen- 
tation into an environment. This prototype is an early attempt to provide insights on how a sophisticated audio processing tool, used in a resource constraint environment provide the efficient functionality and information enriched analytics in an unobtrusive way to the green building applications.

\section{FUTURE GOAL}

The main goal for implementing the ASN is motivated by our work [7] which is to appear in the Percom 2015 proceedings. In this work we had created an acoustic based energy analytic system where the acoustic events of the individual appliances are investigated to determine the microscopic states of the individual appliances to aid in the energy disaggregation and inference algorithm in presence of multiple appliances. As of now we had to perform staged acoustic data collection due to the lack of easily deployable non-intrusive continuous proper measuring device. We hope ASN prototype will serve the purpose of seamless data collection and fast MFCC computation of the acoustic data which has been analyzed in [7]. In the next step, we plan to implement wireless data transfer in a low power network.

\section{ACKNOWLEDGMENTS}

This work is supported by Constellation Energy, NSF Grant CNS-1344990, and REU Supplement.

\section{REFERENCES}

[1] Raspberry pi gpu. http://www.raspberry-projects.com/pi/ pi-hardware/bcm 2835 .

[2] Raspberry pi model b. http://www.raspberrypi.org/product/ model-b/.

[3] Sound exchange (sox). http://sox.sourceforge.net/.

[4] Super gain microphone. http://www. supercircuits.com/accessories/microphones/ super-high-gain-inline-micro-audio-system-pa3-il\# customer-reviews.

[5] J. Cooley and J. Tukey. An algorithm for the machine calculation of complex fourier series. Mathematics of Computation, 19(90):297-301, 1965.

[6] S. B. Davis and P. Mermelstein. Readings in speech recognition. pages 65-74, 1990.

[7] N. Pathak, M. A. A. H. Khan, and N. Roy. Acoustic based appliance state identifications for fine grained energy analytics. In to appear in Proc. IEEE International Conference on Pervasive Computing and Communications (PerCom), 2015. 